\title{
Searching for planets around sdB stars
}

\author{
Andrzej S. Baran ${ }^{1}$, Szymon Bachulski ${ }^{1}$ and Małgorzata Curyło ${ }^{2}$ \\ ${ }^{1}$ Uniwersytet Pedagogiczny w Krakowie, \\ ul. Podchorazych 2, 30-084 Krakow, Poland \\ ${ }^{2}$ Jagielonian University \\ ul. Orla 171, 30-244 Krakow, Poland
}

\begin{abstract}
We present the results of our search for planets around subdwarf B stars. We look for wobbles of the sdB stars seen as sinusoidal variations of the pulsation periods. For a p-mode dominated pulsating sdB star we did not find any signatures of a companion and we marked KIC 10139564 to be a single star. In the case of the g-mode dominated objects the method turned out to inefficient not leading to any conclusion on the planet existence.
\end{abstract}

Keywords. subdwarfs, pulsating stars, exoplanets

\section{Introduction}

One of the evolutionary channels leading to the subdwarf B ( $\mathrm{sdB})$ stars require a companion which helps to strip of the hydrogen envelope from the sdB star. The companion may be another star or a planet. Surveys taken thus far revealed that about $50 \% \mathrm{sdB}$ stars reside in binaries. There are also several objects harboring planets (Table 1). In this work we undertook an effort to look for other sdB stars with planets. We selected a number of pulsating sdB stars (sdBV) observed by the Kepler spacecraft. We analyzed stability of the periods of the pulsation modes which may bear a signature of the Rømer effect. This effect may uncover a presence of a companion. One star in our sample KIC 10139564 is p-mode dominated while the other stars, KIC 2438324, KIC 2991403, KIC 7668647, KIC 3527751, KIC 10670103 pulsate mostly in g-modes.

\section{Methodology}

Pulsation modes can be used to look for companions to the host stars. If a star is orbiting a companion the periods of the pulsation modes are modulated due to the Rømer delay. To measure such modulation we sliced the data into 300-day bins with two adjacent bins overlapping by $20 \%$. This bin size was chosen to minimize uncertainties of the sinusoidal fit which was performed to derive the phase of a sinusoid and resolve close frequencies which could cause beating. The period of the pulsation mode was kept fixed in each fit and adopted from the solution obtained from the analysis of all data. Finally, we calculated the Observed minus Calculated (O-C) diagram from the times of flux maxima to verify stability of the pulsation periods.

\section{Data}

We used short cadence data collected with the Kepler spacecraft. These stars were continuously monitored for over 12 quarters (three years of data). This limits our ability to detect companions in orbits shorter than the data coverage. We downloaded PDC fluxes from the Mikulski Archive for Space Telescope database, $4 \sigma$ clipped and stitched 



Figure 1. The O-C diagrams of four selected modes in KIC 10139564.

Table 1. The list of sdB stars with planets. See text for details.

\begin{tabular}{|c|c|c|}
\hline name & |\# of planet & tool used \\
\hline V391 Peg & 1 & |O-C, pulsations | Silvotti et al. 2007 \\
\hline HW Vir & 2 & | O-C, eclipses | \\
\hline NY Vir & $1+1 ?$ & | O-C, eclipses | \\
\hline | NSVS 14256825 & 2 & | O-C, eclipses | Almeida et al. 2013 \\
\hline | $2 \mathrm{M} 1938+4603$ & | & | O-C, eclipses | Baran et al. 2015 \\
\hline | KIC 5807616 & 2 & | reflection effect | Charpinet et al. $2011 \mid$ \\
\hline KIC 10001893 & | & | reflection effect | Silvotti et al. 2014 \\
\hline
\end{tabular}
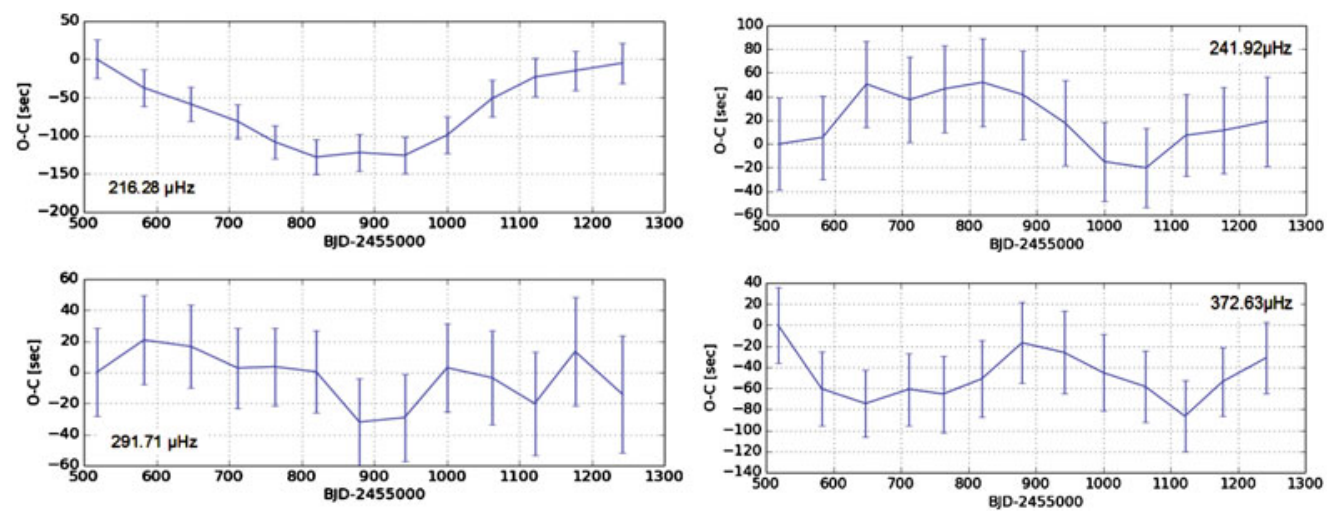

Figure 2. The O-C diagrams of four selected modes in KIC 2438324.

individual months. Next, we calculated the amplitude spectra and selected a few modes with relatively high and stable amplitudes.

\section{Results}

Short-period modes (p-modes in sdBV stars) are excellent tools for probing the O-C diagrams since they allow us to chunk data into narrow bins still covering many pulsation cycles. In addition, the amplitudes of p-modes are usually high enough to obtain precision 

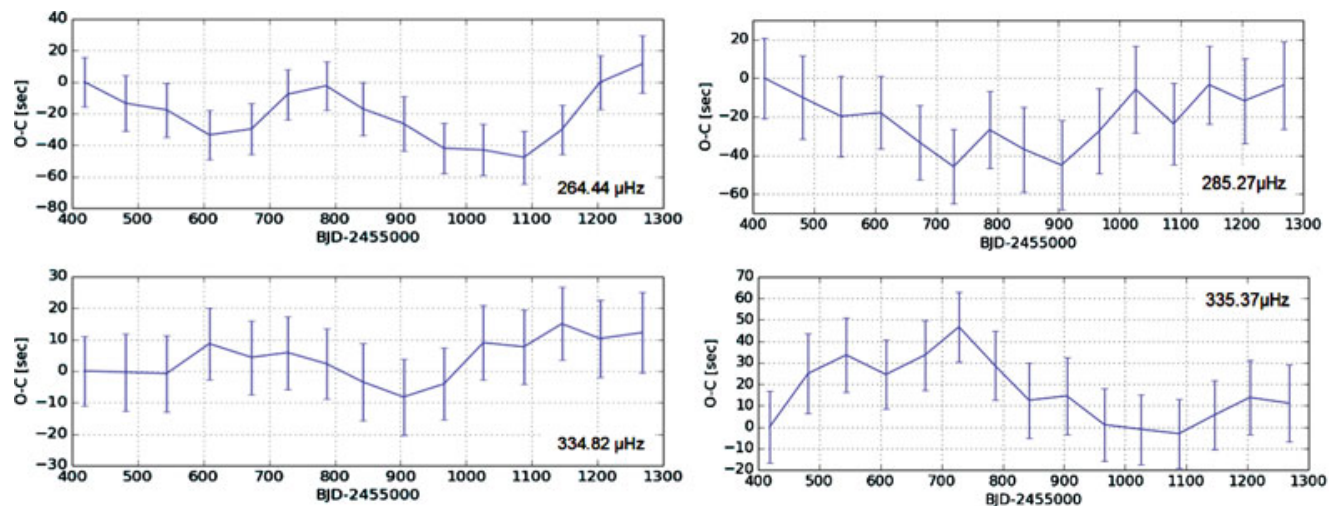

Figure 3. The O-C diagrams of four selected modes in KIC 2991403.
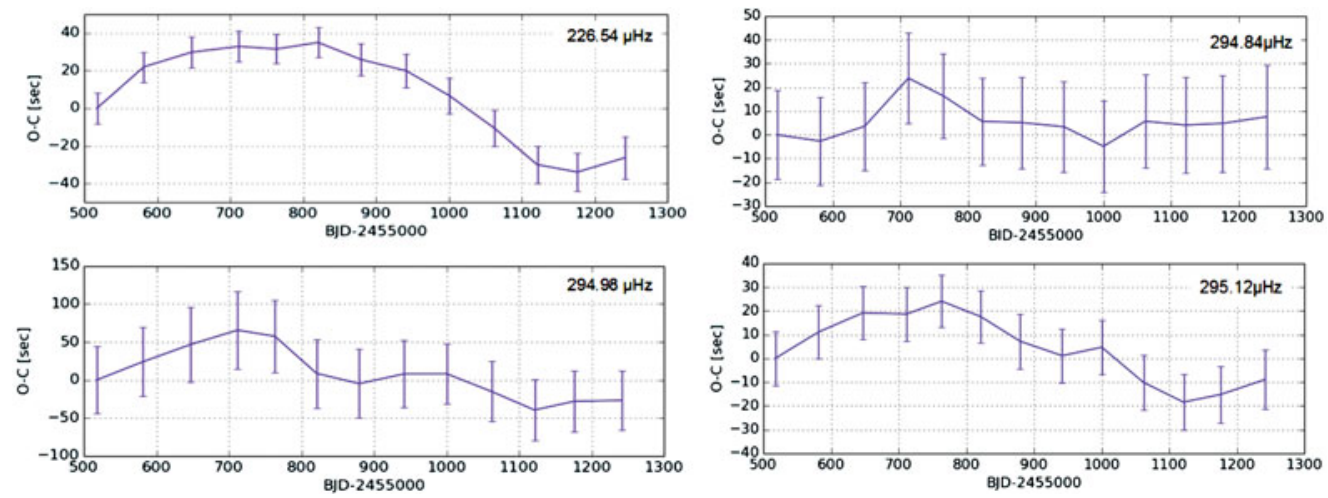

Figure 4. The O-C diagrams of four selected modes in KIC 7668647.
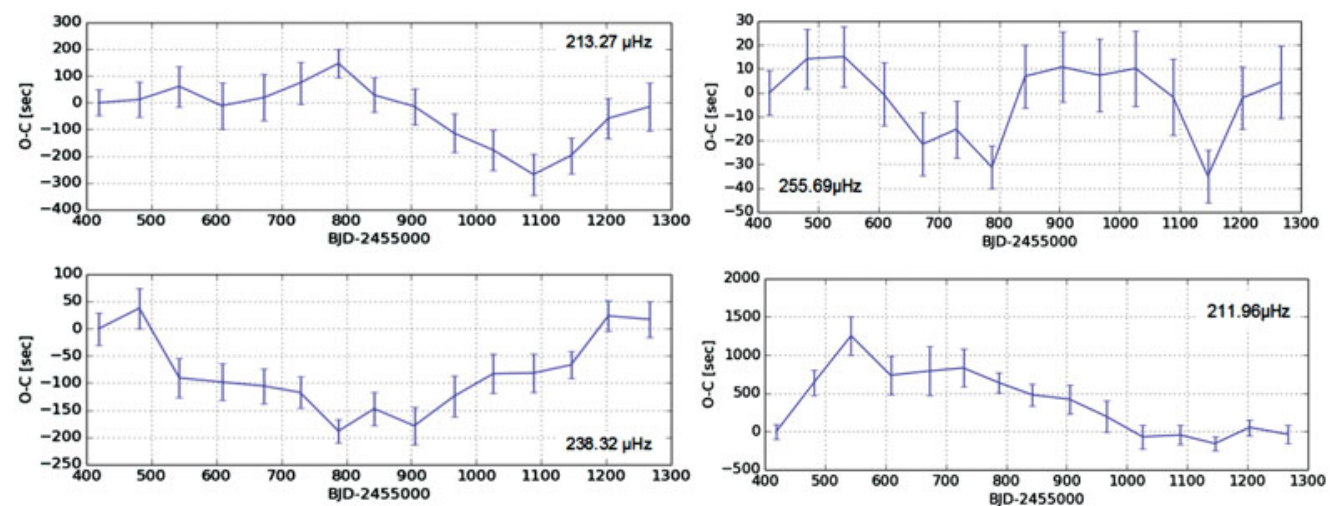

Figure 5. The O-C diagrams of four selected modes in KIC 3527751.

in the O-C diagrams below 1sec. KIC 10139564 is the only p-mode dominated sdBV star among all KIC stars. Figure 1 shows the O-C diagrams of the four selected modes down to a second precision. The periods vary significantly but they do not show consistency between modes which is expected if the pulsation periods are influenced by a companion. Therefore, we conclude that the amplitudes vary intrinsically and we can exclude small mass bodies down to the Jovian mass in orbits shorter than three years. 

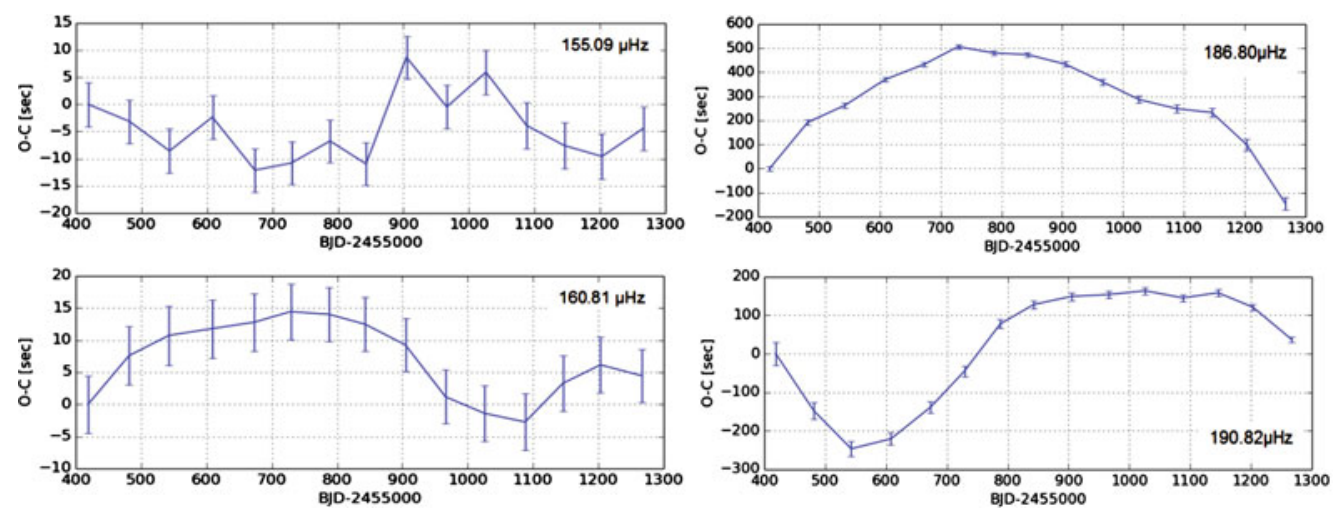

Figure 6. The O-C diagrams of four selected modes in KIC 10670103.

The other five stars presented are g-mode dominated pulsators. The periods of g-modes are hours long and we have fewer cycles in individual bins. In addition, they are of low amplitudes causing derivation of pulsation phases to be less accurate. The $\mathrm{O}-\mathrm{C}$ diagrams of these stars show a few tens of seconds precision, therefore we are unable to find lowmass bodies. Figures 2-6 present the O-C diagrams for the four most suitable modes for each object.

\section{Conclusions}

Our work shows that even the Kepler spacecraft has limitation and cannot overcome precision issues in the $\mathrm{O}-\mathrm{C}$ diagrams for g-mode dominated sdBV stars. Only p-modes are short enough to achieve seconds precisions in the O-C diagrams. We excluded a possibility that KIC 10139564 harbors a planet while we placed no constraints for the g-mode dominated sdB stars. Therefore, we did not increase the number of sdB stars with planets and we show the current list of them in Table 1. We included only planets around KIC stars (regardless of the detection method) or those confirmed from the O-C analysis using either pulsations or eclipses.

\section{References}

Almeida, L. A., Jablonski, F., \& Rodrigues, C. V. 2013, ApJ, 766, 11

Baran, A. S., Zola, S., Blokesz, A., Østensen, R. H., \& Silvotti, R. 2015, A\& A, 577, 146

Charpinet, S. et al. 2011, Nature, 480, 496

Lee, J. W. et al. 2009, AJ, 137, 3181

Lee, J. W., Hinse, T. C., Youn, J-H., \& Han, W. 2014, MNRAS, 445, 2331

Silvotti, R. et al. 2007, Nature, 449, 189

Silvotti, R. et al. 2014, A\& $A, 570,130$ 